\title{
The Victorian Time Signal Service
}

Simon Moorhead

Ericsson Australia \& New Zealand

Summary: A 1945 technical paper from the Telecommunications Journal of Australia is revisited that describes the timing and signalling equipment used at the Melbourne Observatory between 1853 and 1945. The equipment was used to provide the Victorian Time Signal Service for over ninety years.

\section{Introduction}

The measurement of time has a long history stretching back thousands of years. In most ancient civilisations the sun and the moon were observed to determine the time of day, the lunar cycle and the seasons. This led to astronomical observatories being the traditional sources of time measurement.

Fast forward to the Victorian gold rush: the Melbourne Observatory was established at Williamstown in 1853 to provide an astronomical capability for the new colony and a time ball tower for shipping (Cannon 1945). It was then moved to South Yarra in 1864 because of the deterioration of observing conditions at its previous site due to smoke and dust. The Observatory site still exists in South Yarra today and is managed by the Royal Botanic Gardens. (

The Observatory closed in 1945 due to encroaching light pollution from the city. (Wikipedia 2014a) The time signal service was transferred to the Postmaster General's Department (PMG) and the measurement equipment transferred to the PMG Research Laboratories. (Melbourne Museum 2014a)

The TJA paper describes in detail the timing and signalling equipment in the observatory at the time of its closure in 1945, including the pendulum reference clocks and the transit telescope. The paper also discusses the electro-mechanical equipment used to insert the time signal pips into the time signals.

One could be forgiven for assuming the pendulum clocks would lack accuracy compared to today's quartz clocks. These pendulum clocks were actually designed by British railway engineer William Hamilton Shortt in collaboration with horologist Frank Hope-Jones, and manufactured by the Synchronome Co. Ltd. of London, UK. They were the most accurate pendulum clocks ever commercially produced, and became the highest standard for 
timekeeping between the 1920 s and the 1940s. Recent non-intrusive optical testing has verified their error rate to be only one second in 12 years. (Wikipedia 2014b)

Naturally, special precautions were taken in 1945 to ensure the time references were not disturbed in the transfer from the Observatory to the PMG. The PMG also developed new electronic equipment (rather than mechanical mechanisms) to increase the reliability of the pendulum clock references and create the time signal pips.

The transfer of the time signal service to the PMG in 1945 was logical, given the PMG had the means to distribute time references throughout their national telephone network and via high frequency broadcast stations like VNG Lyndhurst in Victoria. Accurate time references have always been required by professionals such as surveyors, for pinpointing locations in remote Australia.

Eventually the quartz clock replaced the pendulum and later the atomic clock (either caesium or rubidium) replaced the quartz clock as the primary standard. Today, GPS is the most practicable time standard in locations with satellite visibility and is linked to US Naval Research Laboratory's atomic standards. The Precision Time Protocol (PTP), described in IEEE standard 1588, is used for Ethernet based time references and is linked to similar atomic standards. (Wikipedia 2014c)

The oldest Shortt pendulum clock (Number 5) from the Melbourne Observatory was donated by the PMG to Museum Victoria in 1972. (Melbourne Museum 2014b)

\section{References}

Cannon, A.H. 1945. 'The Victorian Time Signal Service', Telecommunications Journal of Australia, June 1945, Vol. 5, No. 4, pp. 215-222.

Melbourne Museum-1, 2014a 'Melbourne Observatory \& Standard Time in Victoria' at http://museumvictoria.com.au/collections/themes/1632/melbourne-observatory-standardtime-in-victoria

Melbourne Museum-2, 2014b 'Free Pendulum Clock - William Shortt \& Synchronome Co, London, No. 5, 1925' at http://museumvictoria.com.au/collections/items/407878/freependulum-clock-william-shortt-synchronome-co-london-no-5-1925

RBG, 2014. - Royal Botanic Gardens Melbourne 2014 'Melbourne Observatory' at http://www.rbg.vic.gov.au/visit-melbourne/attractions/melbourne-observatory

Wikipedia-1, 2014a. 'Melbourne Observatory', at http://en.wikipedia.org/wiki/Melbourne_Observatory

Wikipedia -2, 2014b. 'Shortt-Synchronome clock', at http://en.wikipedia.org/wiki/ShorttSynchronome_clock

Wikipedia-3, 2014c. 'Precision Time Protocol', at http://en.wikipedia.org/wiki/Precision_Time_Protocol 


\title{
The historical paper
}

\author{
June, 1945 THE TELECOMMUNICATION \\ Lamb - The Dynamical Theory of Sound; \\ Morse-Vibration and Sound. \\ (3) M a s o n-Electromechanical Transducers and \\ Wave Filters. \\ (4) Shepardson-Telephone Apparatus, p. 55. \\ (5) Wente and Thuras-Moving Coil Telephone \\ Receivers and Microphones, Bell System Technical \\ Journal, Oct., 1931 , p. 565 .
}

(6) Martin and Gray-Master Reference System for

\author{
Telephone Transmission, B.S.T.J., July, 1927. \\ (7) Wall-Applied Magnetism. \\ (8) Marsh-Alloys of Iron and Nickel, Vol. I. \\ (9) Ballantine-Receiver Testing with an Artificial \\ Ear, Electronics, June, 1940.
}

(10) Fletcher-Speech and Hearing.

Reference indices in the appendices relate to the numbers against the items in the Bibliography.

\section{THE VICTORIAN TIME SIGNAL SERVICE}

A. H. Cannon, B.E.E.

Summary: This article describes in some detail the time service given by, and the timing and signalling equipment used at, the Melbourne $\mathrm{Ob}$ servatory until its Time Department closed down on 19th June, 1945. This equipment is being taken over as a going concern by the PostmasterGeneral's Department to ensure continuity of the service. As soon as possible new equipment will be provided, using modern apparatus with which the operating staff of the Department is more familiar.

Introduction: The equipment used at the Melbourne Observatory to provide the Victorian Time Signal Service was built up over many years under the direction of four Government Astronomers, considerable development occurring during the period (from 1915 onwards) when the late Dr. J. M. Baldwin was in charge. With the retirement of Dr. Baldwin in September, 1943, from the State Government service, the responsibility for the Victorian Time Service was taken over by the Commonwealth Government and placed under the control of the Commonwealth Astronomer. In order to provide continuity of service, arrangements were made for the Observatory to carry on with Dr. Baldwin as Officer-in-Charge until a Victorian Time Signal Service could be provided which was based upon the determination of Fundamental Time at the Commonwealth Observatory at Mt. Stromlo, Canberra. Because of the clear atmospheric conditions usually existing at Canberra, and the staff and equipment already at the Observatory, Mt. Stromlo Observatory is well fitted to make Fundamental Time determinations, but it is not practicable for it to originate the actual time signals needed by the State of Victoria. Line rental charges indicated that this should be done by equipment located near the copper centre of Melbourne.

A number of factors led to the PostmasterGeneral's Department being requested to undertake, on behalf of the Commonwejalth Observatory, the generation and distribution of Victorian Time Signals; these factors were the department's technical and distribution facilities, its existing interest in supplying time to the public, and the fact that as portion of its Frequency Standard technique, it had in operation synchronous clocks which were more precise than any of the pendulum master clocks used by Australian Observatories.

The permanent equipment used to provide Time Signals will employ electronic and mechanical components with which operating staff of the Post-office can readily become familiar. However, as the provision of such equipment would take a considerable time, and as it was necessary to close down the Melbourne Observatory Time Service by the middle of 1945 , it was decided to transfer the pendulum clocks time signal equipment from the Melbourne Observatory to the P.M.G. Research Laboratories and to operate the service from there as an interim measure, pending the provision of new equipment which would be operated by the department's State Administration.

This was done, and the first time signals from the Research Laboratories were transmitted at 2.48 p.m. on $19 / 6 / 45$.

Consequently, this article deals with the equipment used at the Melbourne Observatory, together with mention of changes and additions made after installation of the first group of clocks at the Research Laboratories. The proposed new equipment will be the subject of a later article.

Historical: The Melbourne Observatory was first established at Williamstown, opening in July, 1853, with Mr. R. L. Ellery as Astronomer. Because of rapid deterioration in observing conditions (due to smoke and dust), the establishment was moved to its present site on a basalt hillock at South Yarra in 1864, and continued operations from there until June, 1945.

One of the first clocks obtained for the Williamstown Observatory came from England, and is still in service, being used now as the Mean Time "A" clock. Although showing wear in some parts, it still keeps time reasonably accurately, frequently showing changes of rate of less than one second per day.

Until recently the Observatory possessed, as its Master Clocks, two Shortt Free Pendulum Clocks: Shortt No, 5 obtained in 1925 (approx.), and Shortt No. 59, obtained in 1933 (approx.). Clocks of this type have given outstanding performances all over the world, and No. 5 and No. 59 were no exceptions, although No. 5 was noticeably the more stable of the two. 
The other two important clocks used in the time service were the Signalling Clocks, known as the Normal Clock and the Seth Thomas Clock. The former was made at the Observatory soon after it was established at South Yarra, and $\lambda$

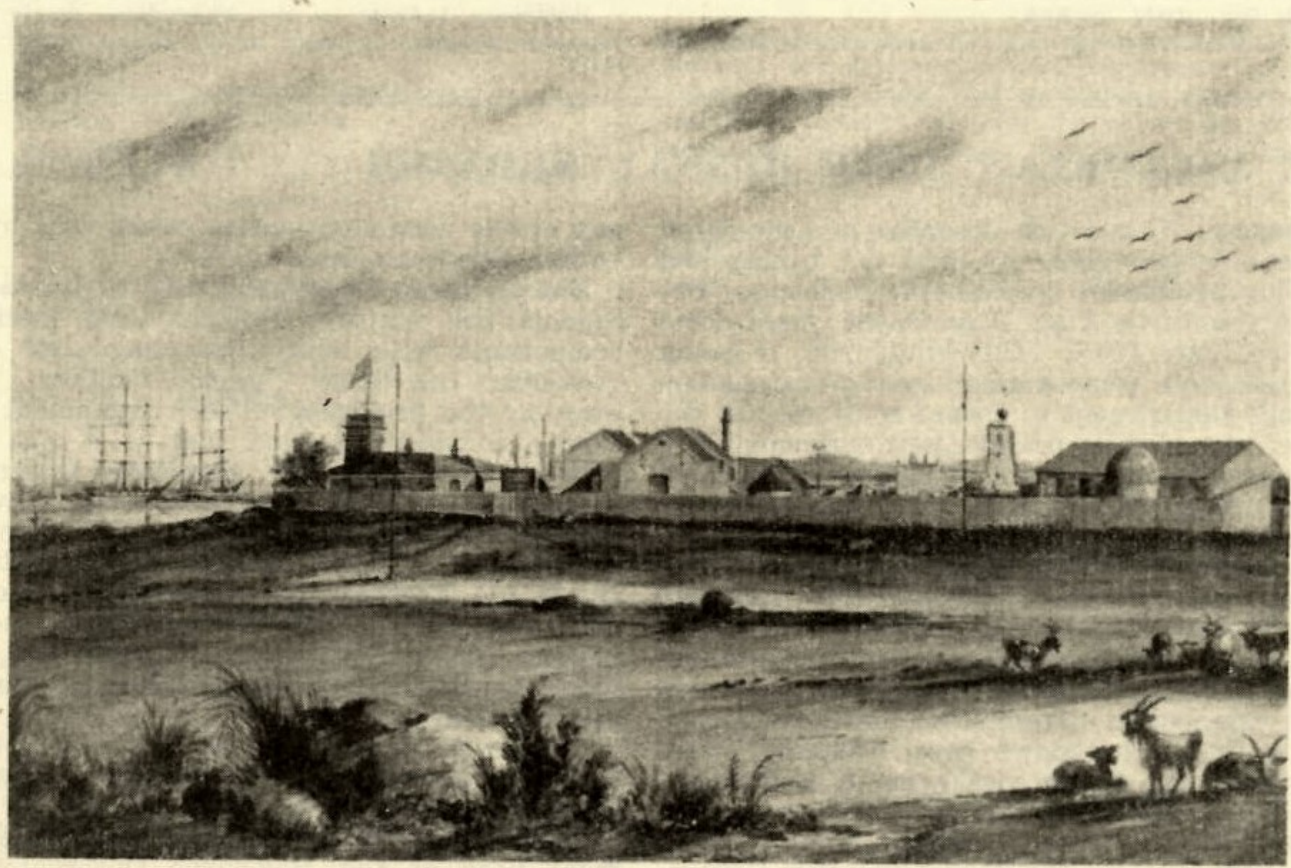

Fig. 1.-The Observatory (Williamstown), 1862.

the latter was purchased from U.S.A. Both are giving good service still, but, of course, their second to second uniformity cannot be compared with that of modern high-grade synchronous electric clocks, such as are controlled by quartz crystal frequency standards.

For some years after 1853, time balls at Williamstown and in the city were the only way in which Observatory time was made public; then the Railways became the first "individual" subscriber to receive time signals, and rapidly the number of subscribers grew to include clockmaking and business firms and Parliament House. At that time power for the electric signalling currents was obtained from large banks of wet Leclanché cells, each subscriber having his own battery at the Observatory.

Gradual progress added such improvements as a special transmitter for marine time signals and a special transmitter for radio time signals, although due to lack of funds the equipment was not modernised as much as desired.

Fundamental Time Determination: By definition, Time is measured in terms of the daily rotation of the earth about its North-South axis, and a "day" is the popular name for the time interval between two successive passages of the sun overhead. Because of various natural causes, of which the most prominent are the elliptical shape of the earth's orbit around the sun and the in- 
moves in a plane which runs North and South, and is perpendicular to the earth's surface; this permits simple checks to determine any small deviation of the actual direction of the telescope from its correct position, and so makes possible the co-ordination of observations on successive nights.

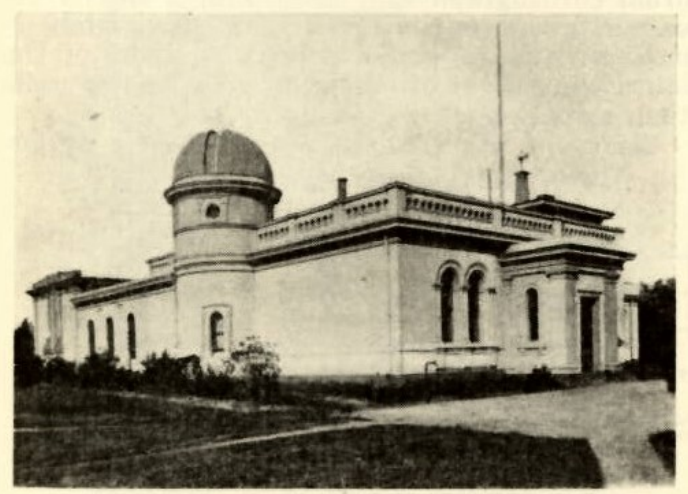

Fig. 2.-The Melbourne Observatory, South Yarra.

Solar and Sidereal Time: Suppose for the moment that we have an accurate clock, capable of ticking at the same rate for a long time. Then if we recorded, from day to day, the times indicated by the clock, when the Sun and also some selected star crossed the North-South plane, perpendicular to the earth's surface, in our locality, we would find that the length of time between successive crossings of the Sun was slightly longer than that between successive crossings of the star. The actual amount is approximately 4 minutes, and after counting 365 solar days, we would find that we had counted 366 star, or sidereal, days, and had returned to our original position.

A clock constructed so that its full cycle (of 24 hours) is equal to the time between two successive passages of the selected star, is called a sidereal (star) clock; the master clocks used by Observatories are usually arranged to keep sidereal time because this is the time used in precision time determinations.

The signalling clocks (e.g., those used to provide time signals for use outside the Observatory) are adjusted to keep Mean Solar Time as accurately as possible, and are usually automatically controlled by the Master Clocks, at infrequent intervals (say, every five or ten minutes). This reduction in the work which the Master Clock has to do considerably improves the timekeeping accuracy.

\section{DETAILS OF THE VICTORIAN TIME SERVICE}

General: As stated above, this service was provided for many years from the Observatory in
South Yarra. Star observations there were made with the eight-inch reversible transit telescope, and were used to check the two Shortt Master Clocks. Associated with each Master Clock was a complete chain of working clocks in order to provide an operating and a stand-by system. These latter clocks were all of the weight-driven, pendulum type, with dead beat escapements. In the following discussion mention will only be made of the working group of clocks.

Types of Time Signals: Four different types of signals were provided:

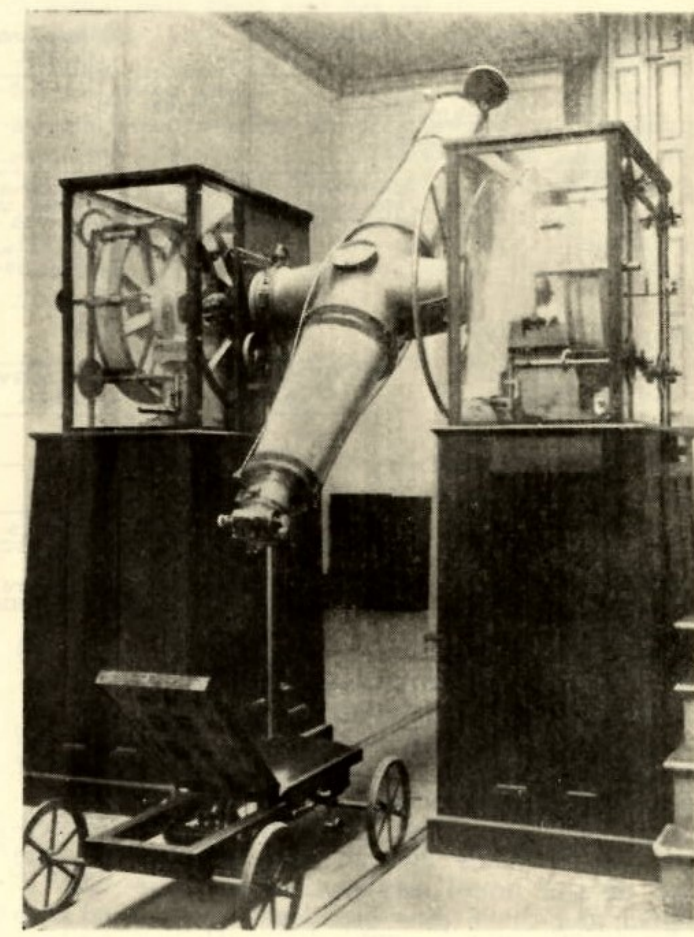

Fig. 3.-The Transit Telescope (the Observatory,
Melbourne).

(1) The "XNG," or International Marine navigation time signal. This signal lasted for three minutes, ending precisely at the hour; during the 57th minute a series of " $X$ " morse code signals, for identification, were generated, followed by six timing pips, the last pip of which occurred at the end of the minute; during the 58th and 59th minutes similar signals occurred, except that "N" signals and then "G" signals replaced the " $\mathrm{X}$ " signals of the 57 th minute.

(2) The "6 pips" broadcast time signal, at the end of each hour, which consisted of a warning pip at 40 seconds, another at 50 seconds, and six timing pips at the end of the 55th, 56th, 
57 th, 58th, 59th and 60th seconds (the last pip again marking the exact hour).

(3) The " 6 pips" signal of (2), repeated once per minute; these signals were used for precision civil timekeeping by the Railways Department, and by some watchmakers.

(4) Pips each second, excepting an omission for identification on the 3rd second; these were used by the Postmaster-General's Department to assist checking the Primary Frequency Standard.

Time Signal Generation Equipment: Four clocks were used to provide these signals: charged batteries, and a plug type single line switchboard was used for testing and for routine comparisons of the various clocks. The main auxiliary equipment consisted of a single pen weight driven drum chronograph, a high-speed tape chronograph and electronic switch, an accurate chronometer, and a radio receiver. The drum chronograph was fitted with a special governor, giving a pen speed of approximately $\frac{3}{4}-$ inch per minute, and was used for most of the inter-comparisons of observatory clocks and radio time signals.

Clock and other relays were generally of low

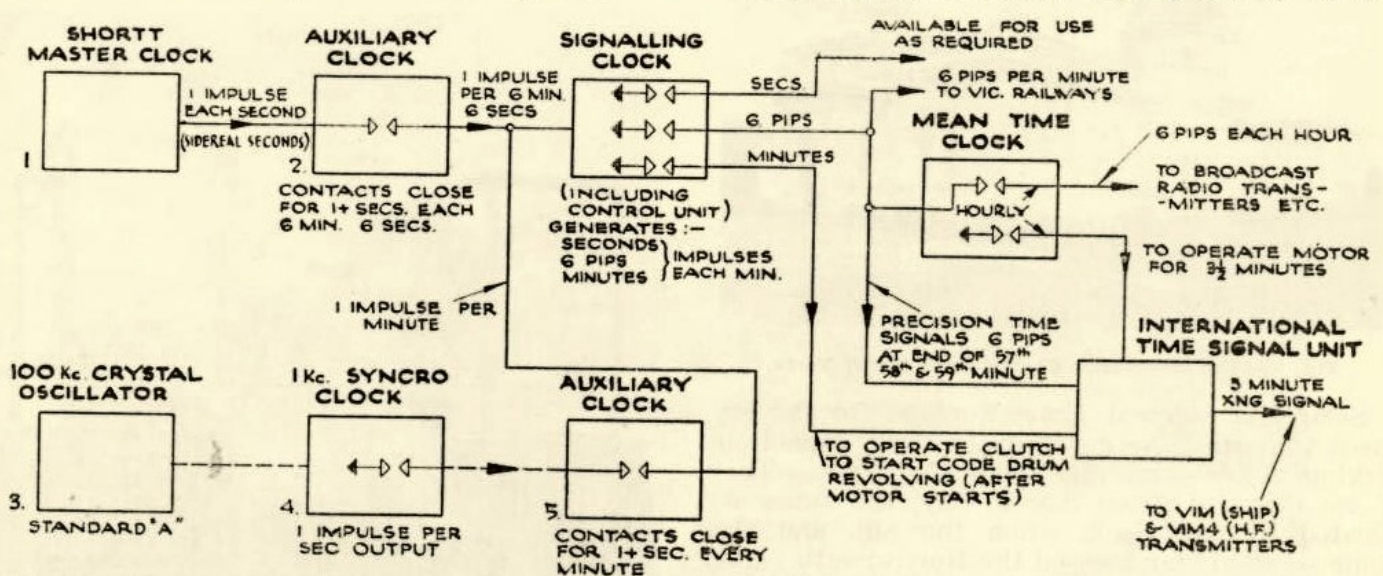

Units 1 and 2 were at the Observatory. They are now re nlaced by Units 3,4 and 5 at P.M.G. Research Laboratories.

(1) The "Shortt" Master Clock; this consisted of two separate units-the "Free Pendulum" and the "Slave Pendulum."

(2) The Signalling Clock, which generated al the actual time signals, repeating each cycle of signals once per minute.

(3) The Mean Time Clock, which selected certain of the time signals (e.g., those near the end of the hour) as required for the "XNG" signal and the 6 pips broadcast signal, and also performed certain auxiliary operations.

(4) The Auxiliary Clock, which selected the time interval between two consecutive corrections of the Signalling Clock by the Master Clock.

The Master Clock kept Sidereal Time, the Signalling Clock and the Mean Time Clock kept Mean Solar Time, and the Auxiliary Clock ran at a rate approximating Mean Solar Time, but adjusted to perform its correction function correctly.

In addition, a motor driven drum carrying raised projections on its surface, corresponding to the code XNG, and fitted with numerous auxiliary switches and relays, was used to generate the identification portion of the navigation signals.

Miscellaneous Equipment: Power was supplied by two sets of six volt 60 amp hour trickle resistance, drawing currents of the order of half an ampere, resulting in operating times estimated as a few milliseconds.

DETAILED DESCRIPTION OF EQUIPMENT

Shortt Master Clock: The aim of the designer, in a clock of this nature, is to make the pull of gravity the sole factor affecting its swing. Disturbing factors are of two kinds-those which are, to some extent, necessary, e.g., the driving force necessary to keep the pendulum swinging, and those which are unnecessary, e.g., changes of temperature.

The outstanding feature of the Shortt "Free Pendulum" Master Clock is the ingenious way in which the Free Pendulum driving impulse is initiated; this is done by the use of another Pendulum, the slave, so that the master, or free, pendulum is only interfered with very slightly. As well as this all possible refinements are incorporated in the master pendulum.

In detail, the master clock is composed of two separate clocks, known as the Free Pendulum Clock and the Slave Clock. Both keep very closely in step, and are adjusted to keep (usually) sidereal time. The Free Pendulum is usually mounted on rock in a location free from vibration and from earth movements which 
might affect the value of the constant of gravitation. Although the pendulum is temperature compensated, some care is taken to keep the

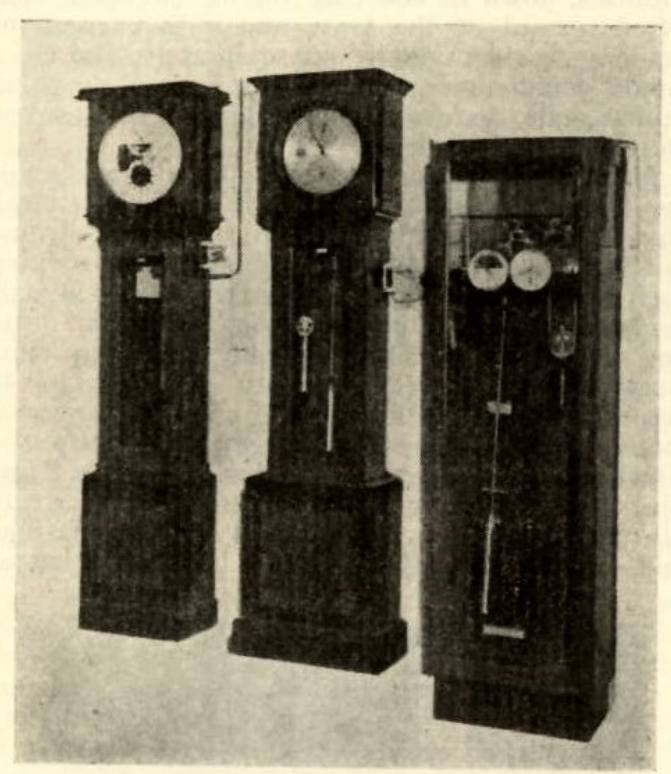

Fig. 5.-Time Signal Clocks, P.M.G. Research Iaboratories. Ieft to right: Mean Time Clock, Auxillary Clock,

room, or vault, temperature as steady as possible. The pendulum itself swings in a constant pressure cylinder, the air pressure in which is adjusted to be only a few $\mathrm{cm}$. of mercury, as this gives the best balance between certain residual effects. Because of the low pressure only a small driving force is required, and the mechanism is arranged so that this is given relatively seldom and in a gradual manner, at the centre of the swing of the pendulum, so as to cause a minimum of interference with the evenness of its oscillation. This is the only unavoidable disturbance which the Free Pendulum suffers because the work of counting the time duration between successive applications of the driving force, and of tripping the mechanism to apply the driving force, is done by the Slave Pendulum.

The Free Pendulum is driven by a small weight applied by the jewel $R$ on to the wheel $\mathrm{J}$. attached to the pendulum rod; as $\mathrm{R}$ drops it first pushes the free pendulum sideways in the direction of its motion at that instant, and then drops clear of the inclined surface to trip a catch which closes a contact in the electrical circuit used to transmit a synchronising impulse to the Slave pendulum. Normally, each second synchronising impulse speeds up the Slave, which would otherwise run slow, so that on the average the Slave keeps correct time. The Slave counts seconds to determine when the jewel driving the Free Pendulum shall be released again, and also supplies the outside time signals (via other clocks, of course). The Slave is a robust, high-quality clock self-driven by the small roller which runs down the inclined plane on the arm attached to the pendulum rod. It is usually mounted in an easily accessible location and its routine maintenance requires the control of its own rate (i.e., between successive synchronising impulses from the free pendulum), by the adjustment of small weights on its bob, so that consecutive synchronising impulses alternatively miss and act on it.

As the Free Pendulum is impulsed every 30 seconds, the Slave clock is only required to run so that its error is small over a time of 60 seconds, since after that time the synchronising impulse from the free pendulum will adjust the time error in the Slave by any amount necessary. So the ideal driving conditions for the free pendulum, of a uniform force applied for uniform times at uniform time intervals, are met almost perfectly.

Before the Shortt "Free" pendulum was invented the non-uniformity of driving conditions

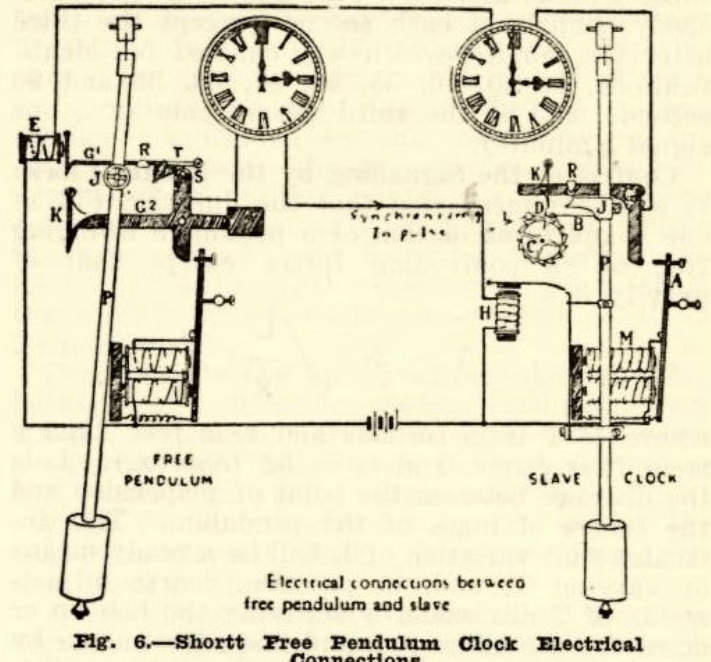

was the major obstacle to more precise timekeeping. For many years after its introduction in 1921 the Shortt clock was unrivalled, and competitors and technique were so backward that it was hard to analyse its imperfections. With the advent of more precise "Crystal Clocks," which form portion of Frequency Standards, a more accurate clock and means for measuring short period (second to second or day to day) irregularities of the Shortt have become available, and work is going on in England, at least, to improve the Shortt clock still further. As it is possible that this will result in a considerable increase in accuracy, and because of 
the simplicity (and hence reliability) of the pendulum clock, this work is of some importance. However, further discussion of this point is outside the scope of this paper.

Because the Free Pendulum is constructed to be independent, as far as possible, of outside forces, difficulty is usually experienced in changing its rate occasionally to compensate for the slight amount of ageing which occurs. This was done, in the case of the Victorian Observatory, by a small permanent magnet, under the bottom of the evacuated case, which interacted with the steel pendulum rod; the range of control was approximately one second per day.

The Signalling Clock: This was a high-grade pendulum clock, weight-driven through a dead beat escapement; it was adjusted to keep Mean Solar Time, and generated the actual time signals. It was controlled by the Shortt Master Clock, the correction impulses occurring each six minutes six seconds (Solar).

All the time signalling was done by arms carrying electrical contacts, which were operated by teeth on wheels mounted on the shaft rotating once per minute. There were three of these wheels, and they were cut to give, respectively, signals at each second (except the third after the minute, which was omitted for identification), at $40,50,55,56,57,58,59$ and 60 seconds, and at the third second only (i.e., one signal a minute).

Control of the Signalling by the Master Clock: It will be remembered that the duration ( $T$ ) of one complete oscillation of a pendulum swinging free of all controlling forces except that of gravity is

$$
\mathrm{T}=2 \pi \quad \sqrt{\frac{\mathrm{L}}{\mathrm{g}}}
$$

where, if $\mathrm{T}$ is in seconds and $\mathrm{L}$ is feet, then $\mathrm{g}$ is in ft./sec./sec. and is $=32$ (approx.). L is the distance between the point of suspension and the centre of mass of the pendulum. This indicates that variation of $\mathrm{L}$ will be a ready means of varying $\mathrm{T}$, and, in practice, coarse adjustments of $\mathrm{T}$ are made by screwing the bob up or down the pendulum rod, and fine adjustments by adding weights on the top of the bob; as this latter step effectively raises the centre of mass of the pendulum and so decreases L, any added weight will decrease the length of the period of oscillation and so make the clock run fast.

At the Melbourne Observatory, control of the signalling clock, by the master clock, was obtained by the use of mechanism which caused a small weight to be put on or lifted off a tray attached securely to the pendulum rod. The clock was adjusted by varying the small weights on the top of the pendulum bob so that, with the control weight off, it ran slow by approximately $\frac{3}{4}$ second a day, and the size of the weight was chosen to make it gain approxi- mately $\frac{3}{4}$ second a day when the weight was on. With these adjustments made correctly, the control weight was, over a period of, say, 61 minutes, down in the tray for as long as it was lifted up out of the tray, and with corrections made each six minutes, approximately, the clock would usually gain, or lose, approximately three milliseconds, before the position of the control weight was altered.

Mean Time Clock: As well as providing signals for the XNG unit as described later, this clock was used to eliminate, for broadcast transmitters, 59 of the 60 "six pip" signals generated each hour by the signalling clock. As the signals to be transmitted occurred on the 40th, 50th, and then 55th to 60th seconds, the Mean Time clock contacts concerned were closed from 45 seconds before the hour to 15 seconds (approximately) after. These requirements specify the timekeeping precision required of the Mean Time Clock; to provide a factor of safety, time errors of more than five seconds are not permissible, and, as the rate of the clock is usually better

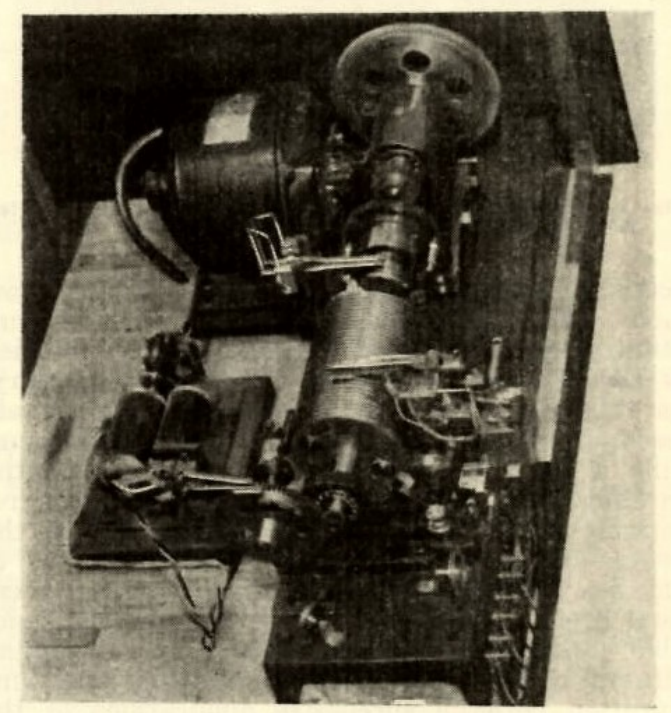

Fig. 7--International Time Signal or XNG Unit
(10oking down), P.M.G. Research Laboratories.

than one second per day, a reasonable margin is available for long week-ends, holidays, etc.

Auxiliary Clock: This clock carried one pair of make contacts which were closed each six minutes six seconds for approximately one and a quarter seconds, during which time a control impulse from the master clock was permitted to pass to the correction unit of the signalling clock.

XNG Transmitter: This unit, under the control of the signalling clock, generated the identification portion of the XNG signal; the time sig- 
nal portion of the XNG signal (viz, the six pips at the end of each minute) was transmitted direct from the signalling clock to obtain the highest possible accuracy.

Driven by a synchronous motor through a magnetic clutch, was the programme drum (approximately $6 \mathrm{in}$. long and $3 \frac{1}{2}$ in. diameter) which carried the XNG signals in the form of raised projections on its cylindrical surface; as required at the start of a run, a pair of electrical contact carrying arms were released by an electro-magnet to cause one end of the lower arm to trail on the drum surface so that it was raised by each projection sufficiently to close the two contacts. A deep spiral thread ( 6 turns per inch) was cut into the surface of the drum, running in between the signalling projections, and into this was fitted a hardened steel tongue which was fastened on to the base of a carriage which carried the two contact arms mentioned above; consequently, when the drum rotated, the contact making arms were moved parallel to the main axis of the drum, so that the arms passed over each signalling projection in turn. On this contact arm carriage was also mounted, at the forward end, a trip mechanism to open the crrcuit of the magnetic clutch when the contact arms reached the end of the XNG programme; while at the rear end of the contact arm carriage was a spring-loaded projection which, when the carriage had almost completed its return stroke, caused a spring steel finger to swing out and, by engaging a projection on the rear end of the programme drum, stop its revolution without excessive shock.

The full programme lasted practically three minutes, and near the end of each minute the time signal output of the unit was switched from the drum contact arms to take the six pins from the output of the signalling clock by a cam operated from main drum drive shaft.

The induction motor was started at 3 minutes 14 seconds before the hour, and stopped 15 seconds after the hour by relays controlled from an hourly contact (lasting for three and a half minutes) on the Mean Time Clock; while the magnetic clutch was started at 2 minutes 57 seconds before the hour by the minute signal on the signalling clock. Use of the correct minute signal was ensured by keeping its circuit open until the Mean Time Clock hourly signal occurred. The first minute impulse after this circuit became closed locked un the trip mechanism carried at the forward end of the programme arm carriage and so closed the magnetic clutch circuit and held it closed until the trin mechanism was unlocked mechanically by the drum after the programme had been concluded.

As stated above, drive on the forward transmitting portion of the cycle was by electric motor; at the end of the forward portion of the cycle the clutch was de-energised, allowing the drum to be rotated in the opposite direction back to its start position by a torque provided by a weight-loaded cord which had been wound up while time signals were being transmitted. This reverse rotation of the programme drum also returned the contact arm carriage, which was gravity-loaded to assist this movement.

Switching Facilities: In order to permit the maximum use of clocks in the reserve group comprehensive arrangements were made to switch in practically any clock of the reserve group, in place of its corresponding member of the working group. At the same time arrangements were provided to enable the various clocks to be readily inter-compared, using a tape or drum chronograph.

Overall Accuracy of the Equipment: The main factors controlling this, under normal operating conditions, were the deviation of the master clock from its predicted value (obtained by extrapolation based on star observations) and the irregularities in the spacing of the teeth of the transmitting wheels on the seconds shaft.

Star observations were normally attempted each night, but owing to Melbourne weather conditions periods of up to a week between satisfactory observations were common. On many occasions the master clocks would keep time to within, say, 10 milliseconds, over this period, but on other occasions errors of as much as 50 milliseconds were noted. The position was further complicated by the inaccuracy of the star observations (the probable error was usually about 25 milliseconds); this made severa consecutive star observations necessary before the master clock error could be determined reliably. In addition, the spacing of the teeth in the seconds wheels could cause errors of 10 or 20 milliseconds.

Consequently the estimated probable error lay between 20 milliseconds (when conditions were stable) to possibly 60 or 70 milliseconds when conditions were bad. Over the last few years this latter figure was considerably reduced to, say, 20 or 30 milliseconds) by the use of the Post-office Crystal Clock (Frequency Standard) as a third master clock.

Modifications Made When Installing the Time Signalling Equipment at the Research Laboratories: Continuity of operations during the change from Observatory to Post-office operation was obtained by installing the clock group, which had been in reserve at the Observatory, in the Research Laboratories. The master clock of this group was not available, having on August, 1944. been sent to Mt. Stromlo Observatory. At the Research Laboratories, the function of this clock was performed by a synchronous clock driven from one of the crystal oscillators of the Primary Frequency Standard. This gives a greater accuracy and flexibility, at the cost of greater fault liability. This greater fault liability will shortly be practically eliminated by the insertion of a stable pendulum clock between the 
synchronous clock and the signalling clock to carry on as a master clock if the synchronous clock stops. Because the crystal master clock keeps Mean Solar time certain auxiliary equipment was modified to enable the original control system of the Signalling clocks to be maintained.

Some increase in the reliability of the control of the Signalling clock (which had been giving erratic trouble at the Observatory) was obtained by increasing the control weight and also shortening the time between comparisons of the Master and Signalling clocks.

Better use of cable pairs was made by installing splitting relays (for the hourly six pips signal) at City West Exchange.

Use of Frequency Standard Equipment with

the Pendulum Clock Units: Although all details of this aspect have not been finalized, the following notes are included as a matter of interest:

At present the main units of the Frequency Standard are two $100 \mathrm{kC}$ quartz oscillators ("A" and " $\mathrm{B}$ "); held as a stand-by is the previous Frequency Standard, a 1000 c.p.s. Valve Maintained Tuning Fork.

Timing of all three units is effected (using divide by ten multi-vibrators as necessary) by driving 1000 c.p.s. synchronous clocks from portion of the output of the unit concerned.

As well as indicating in a conventional way, hours, minutes and seconds, the one r.p.s. shafts of these clocks produce seconds impulses of high precision (erratic variations are of the order of one millisecond). The phase of these in seconds impulses can be precisely adjusted by moving the back (normally fixed) contact of the pair of seconds contacts, so that the contacts make earlier or later. Seconds Signals generated from a "Crystal" clock operated in this way (from Standard "A" at present) are transmitted twice daily over a normal telephone carrier circuit to Mt. Stromlo Observatory, Canberra. Comparison of these signals at the Observatory, with star observations and radio time signals provides the link between Time Signals generated in Melbourne and Fundamental Time, and also provides one means for calibrating the Frequency Standard.

The precision of this method of time keeping can be gauged from the fact that it is possible to predict ahead, on the basis of past behaviour against Observatory Time Signals, that "Frequency Standard" time after an interval of, say, one month. will be within 20 milliseconds of the corrected Observatory Time Signals at that date. (Corrections are usually not known till some weeks afterwards.)

Seconds impulses generated in this way are converted into electrical power by the use of gas discharge tubes as impulse generators, and the pulses of power, after being lengthened, are fed into the Signalling Clock control circuit in place of signals previously generated by the Slave portion of the Shortt Master Clock.

The phase differences between series of second signals from various sources (e.g., time signals, synchronous or pendulum clocks) are determined in several ways, e.g., by the use of pen or spark tape chronographs, or by adjusting the phase of one series to cause it to coincide with the series of seconds impulses from another source. In the former case, measurements can be made with negligible delay and errors of less than two milliseconds; while in the latter case the delay is also small and the measurement inaccuracy can be made less than one millisecond. In this latter case, coincidence can be observed visually on a Cathode Ray Oscillograph, or audibly by feeding the signals into a special Valve Oscillator/amplifier unit which drives a loud speaker; here coincidence is indicated aurally by a cancellation of one signal by the other, or by their super-position.

A purely electrical method of controlling a weight-driven pendulum is being developed to supersede the lifted-dropped weight method at present used on the signalling clock; such a development is necessary, because in the event of failure of the control signals the control weight remains dropped, thus giving the clock a large gaining rate. The unit being developed determines the phase of the pendulum clock seconds, relative to the control seconds, and converts this phase difference into a unidirectional magnetic force, which operates on a permanent magnet fastened to the bottom of the pendulum bob, and so causes what is, in effect, a change in the value of the constant of gravitation, as far as the period of the pendulum is concerned. This system of control is expected to be especially useful when applied to the Shortt Clock, where the master pendulum swings in an evacuated space, thus rendering impracticable control of its rate by the usual method of adding small weights to, or removing them from, the pendulum bob.

Future Action: It is proposed to instal new equipment, using electronic and telephone apparatus, at City West Exchange as soon as possible, so that the service can be handled by the department's normal operating staff.

Acknowledgment: The author's personal thanks are due to the late Dr. J. M. Baldwin and members of the Observatory staff; and to the officers concerned in the Research Laboratories, for their interest and enthusiasm. 